\title{
Dilemas diagnósticos y terapéuticos en talla baja
}

\author{
MJ. Ceñal González-Fierro \\ Pediatra. Servicio de Pediatría. Hospital de Móstoles. Móstoles, Madrid. España.
}

Rev Pediatr Aten Primaria. 2009; I I Supl I6:s /55-s I 72

María Jesús Ceñal González-Fierro, mjesuscenal@terra.es

\section{Resumen}

Una separación clara entre una talla normal y una talla baja es difícil de establecer. Se consideran tallas altas las situadas entre +2 desviaciones estándar (DE) para la edad, sexo y grupo étnico y tallas bajas las que se encuentran por debajo de $2 D E$, que corresponde aproximadamente al P3 de las curvas de referencia. Se distinguen dos grupos: talla baja normal, en el que están incluidos los niños con talla baja familiar, con retraso constitucional del crecimiento y desarrollo o con retraso de la pubertad; y talla baja secundaria a una causa patológica. Para el diagnóstico es necesario realizar historia clínica, exploración física, valorar el grado de maduración realizando la determinación de la edad ósea y el pronóstico de talla, analítica general, anticuerpos antitransg/utaminasa A, hormonas tiroideas, factores de crecimiento (IGF-1 e IGFBP-3), si es necesario se realizará un estudio de la secreción de hormona de crecimiento (normal > $10 \mathrm{ng} / \mathrm{ml}$ ), cortisol libre urinario, resonancia magnética nuclear craneal de la zona hipotálamo-hipofisaria (hipoplasia de la adenohipófisis con o sin sección del tallo hipofisario, la neurohipófisis ectópica y la silla turca vacía), cariotipo, y/o estudios de genética molecular. El tratamiento de la talla baja secundaria a una patología específica será el de esa patología cuando sea posible. Actualmente las indicaciones de tratamiento de talla baja con hormona de crecimiento autorizadas en España son el déficit de hormona de crecimiento, el síndrome de Turner, la insuficiencia renal crónica, el síndrome de Prader Willi, el crecimiento intrauterino retardado y la alteración del gen $\mathrm{SHOX}$.

Palabras clave: Crecimiento, Talla baja, Curvas de referencia, Enfermedades relacionadas con el crecimiento/diagnóstico, Enfermedades relacionadas con el crecimiento/tratamiento.

\section{Abstract}

A clear separation between a normal height and a short stature is difficult to establish. High height is considered between +2 standard deviations (SD) for age, sex and ethnic group and short stature is considered below $2 S D$, which approximately corresponds to $P 3$ of the reference growth charts. There are two groups: normal short stature in which are included children with familiar short stature, with constitutional delay in growth and development or with delayed puberty and short stature secondary to a pathologic condition. For the diagnosis is necessary to perform clinical history and physical examination, to assess the degree of maturation making identification of the bone age and the prediction of adult height, general analysis, antitransg/utaminase $A$ antibodies, thyroid hormones and growth factors (IGF-1 and IGFBP-3).

La autora declara no presentar conflictos de intereses en relación con la preparación y publicación de este artículo. 
When appropriate, a study of the secretion of growth hormone (normal $>10 \mathrm{ng} / \mathrm{ml}$ ), urinary free cortisol, cranial magnetic resonance imaging of the hypothalamic-pituitary area (hypoplasia of the adenohypophysis with or without section of the pituitary stalk, ectopic neurohypophysis and the empty "sella turcica"), karyotype, and / or molecular genetic studies will be made. The treatment of short stature secondary to a specific pathologic condition will be that of the condition whenever that is possible. At present the indications for treatment of short stature with growth hormone approved in Spain are the growth hormone deficiency, Turner syndrome, chronic renal failure, Prader Willi syndrome, small for gestational age and alteration of the gene Shox.

Key words: Growth, Short stature, Growth charts, Growth disorders/diagnosis, Growth disorders/treatment.

\section{Introducción}

La talla depende de factores genéticos (sexo, raza, talla de los padres) y ambientales (entre los que tiene gran importancia la nutrición). Una separación clara entre lo que es una talla normal y lo que es una talla baja es difícil de establecer. La gran importancia social que se concede en el momento actual a la talla alta, está propiciando un aumento de las consultas por talla baja en pacientes con talla normal, pero por debajo de la media.

\section{Patrón de crecimiento humano}

El crecimiento de la especie humana se caracteriza por presentar dos períodos de crecimiento rápido con sus fases de aceleración y desaceleración, separados por un período de crecimiento estable ${ }^{1}$. El primero de estos períodos de crecimiento acelerado corresponde al fetal y los primeros meses de vida extrauterina (componente fetal-primera infancia) y el segundo al estirón puberal (componente puberal). Entre ambos la velocidad de crecimiento es constante (componente prepuberal o de la segunda infancia), observándose alrededor de los 7 años un ligero incremento que coincide con la adrenarquia (figuras 1 y $2)^{2}$. Existe un claro dimorfismo sexual en el estirón puberal, siendo más precoz y menos intenso en las mujeres que en los varones.

\section{Definición de talla baja}

La talla, como muchas otras variables biológicas, se comporta como una variable continua, sus valores muestran una distribución "normal" que puede representarse como una campana de Gauss (figura 3).

Con criterio estadístico se consideran tallas normales las situadas entre \pm 2 desviaciones estándar (DE) para la edad, sexo y grupo étnico. Son tallas bajas las que se encuentran por debajo de $2 \mathrm{DE}$, 
Figura 1. Componentes del crecimiento. Velocidad de crecimiento. Adaptada de Kalberg.

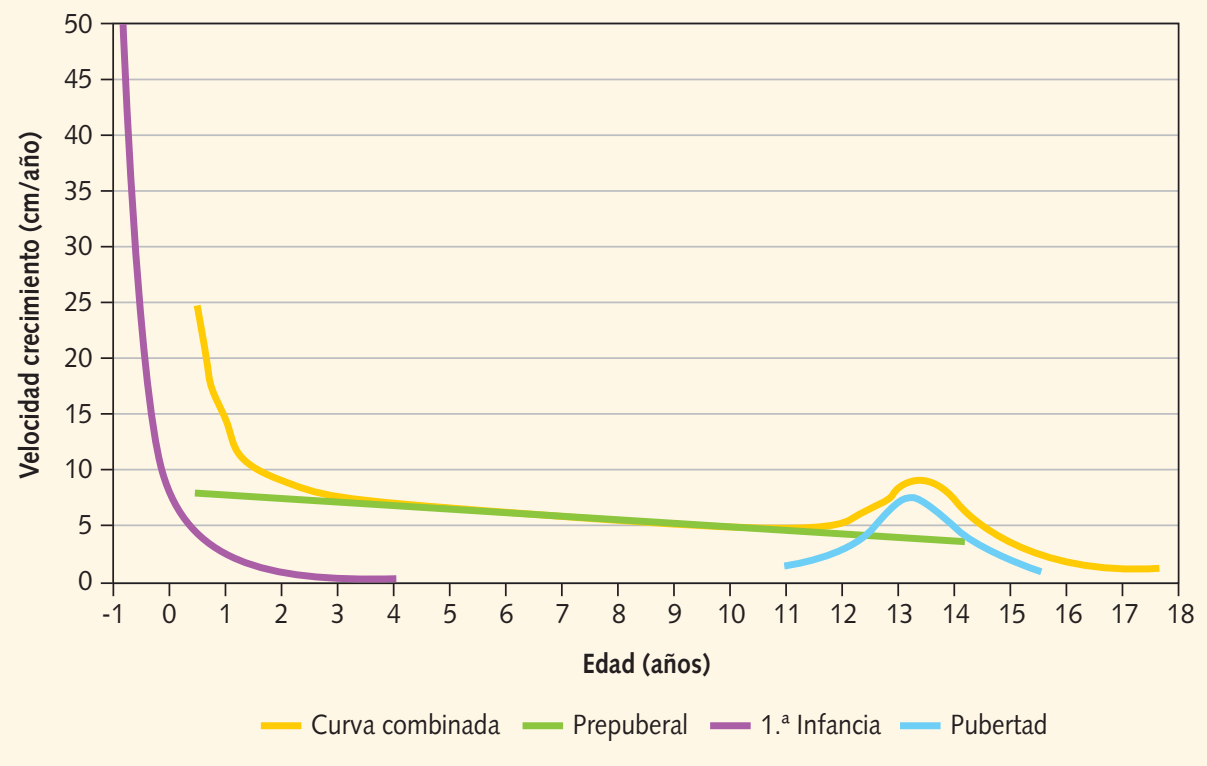

Figura 2. Componentes del crecimiento. Talla alcanzada. Adaptada de Kalberg.

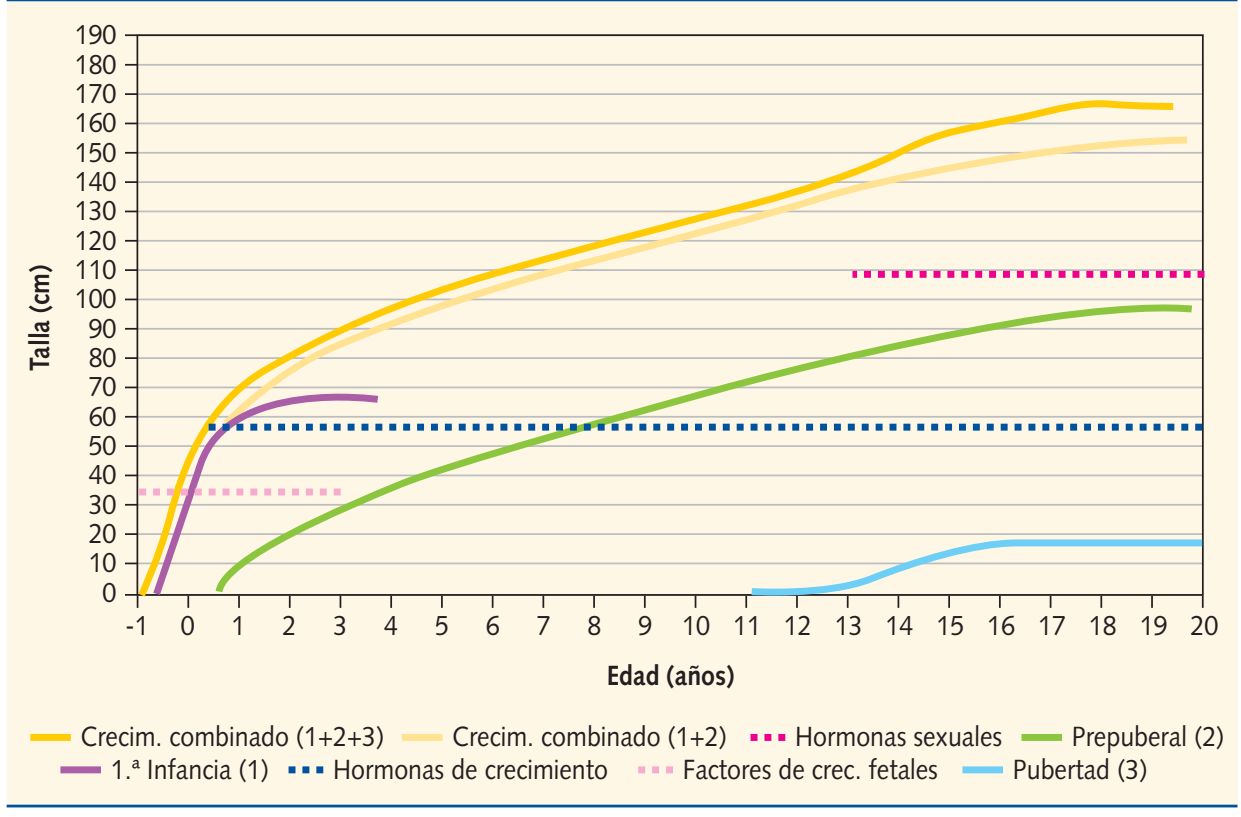


Figura 3. Propiedades de la curva de Gauss.

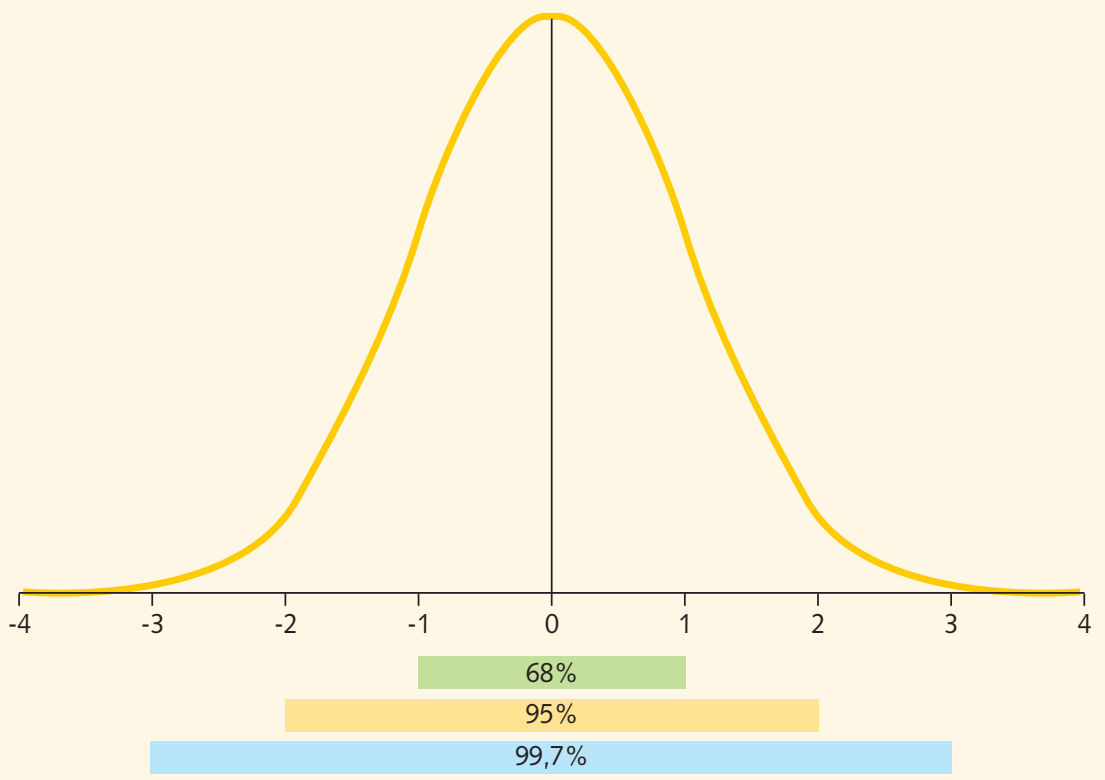

que corresponde aproximadamente al P3 de las curvas de referencia para su edad, sexo y grupo racial. Una talla inferior a -2 DE no implica que sea necesariamente patológica.

También se consideran tallas bajas aquellas por encima de $2 \mathrm{DE}$, pero con un pronóstico de talla adulta más de 2 DE por debajo de su talla diana.

\section{Curvas de referencia}

Se recomienda utilizar las que mejor se adapten a nuestra población. En nuestro medio disponemos de:

- Curvas y tablas de crecimiento. Estudio longitudinal y transversal. Fun- dación Faustino Orbegozo. 2002, Bilbao ${ }^{3}$.

- Estándares longitudinales de niños españoles. Centro Andrea Prader. 2002, Zaragoza 4 .

- Patrones de crecimiento en niños normales. 2003, Barcelona5.

Recientemente se han publicado los resultados del "Estudio transversal español de crecimiento 2008 (Parte II)"6. En este estudio se ha valorado el crecimiento en una población caucásica española procedente de Andalucía, Barcelona, Bilbao y Zaragoza cuyas medidas se han realizado entre los años 2000 y 2004. Las tablas procedentes de este 
estudio ya están disponibles?. Estas tablas, al ser realizadas exclusivamente con población caucásica española, deben ser valoradas respecto a su utilización en población inmigrante, ya que los autores encuentran diferencias al compararlas con otros estudios europeos y americanos. La utilización de tablas realizadas en los países de origen es difícil en la práctica diaria, y además la diferencia de la situación social y nutricional de la población en su país de origen y en el nuestro es un factor que disminuye la validez de su utilización.

La Organización Mundial de la Salud (OMS) ha propiciado un estudio multi- nacional: "Estudio multicentro de las referencias del crecimiento (MGRS, por sus siglas en inglés)" ${ }^{8}$, cuyo objeto es elaborar nuevas referencias de crecimiento para lactantes y niños pequeños (0-5 años). Se pretende crear una sola referencia internacional que represente el crecimiento fisiológico de los niños menores de 5 años y establezca al lactante alimentado al pecho como el modelo normativo de crecimiento y el desarrollo. Existen datos que demuestran que las pautas de crecimiento de los preescolares sanos, bien nutridos en el mundo son similares ${ }^{9,10}$ (figura 4). Sin embargo los autores del "Estudio trans-

Figura 4. Talla media (cm) de 2 a 5 años para cada uno de los seis países.

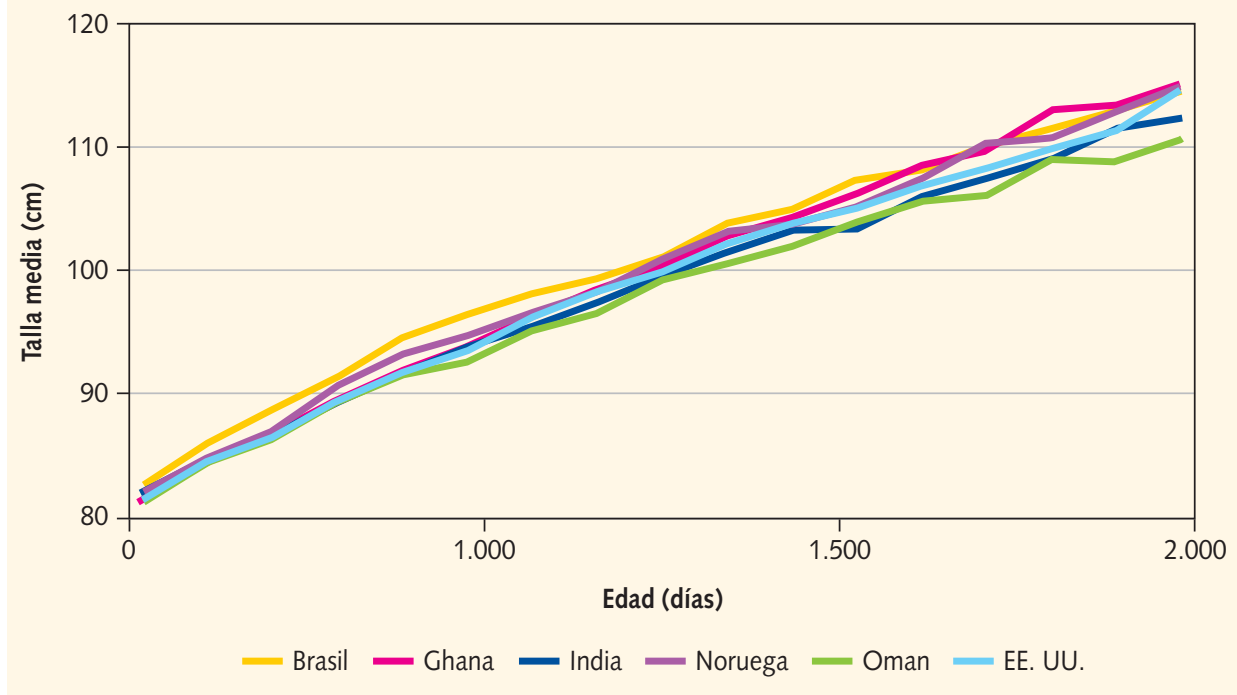

Tomado de WHO Multicentre Growth Reference Study Group (Acta Paediatr Suppl. 2006;450:61). 
versal español de crecimiento 2008"7 encuentran que aunque los valores de talla, peso e índice de masa corporal al nacer son similares a los del estudio de la OMS, a los 5 años de edad estos valores son más altos en la población española.

\section{Velocidad de crecimiento (VC)}

Se expresa en cm/año y se aplica a la edad intermedia entre las edades en que se realizó la observación. El tiempo transcurrido entre las dos mediciones debe ser calculado con exactitud (intervalo decimal), extrapolando el crecimiento obtenido a un año, mediante una regla de tres. El intervalo ideal de observación es de un año (entre 0,881,12 años) y en ningún caso inferior a 6 meses; ya que, el error se incrementaría como resultado de las grandes fluctuaciones que, en períodos más cortos de tiempo, puede experimentar el ritmo de crecimiento ${ }^{11}$. El ejemplo más conocido serían las variaciones estacionales, con mayor crecimiento, en la mayoría de los niños, en primavera y verano ${ }^{12}$.

La interpretación de una VC aislada, como normal o patológica, puede ser difícil debido a su naturaleza cíclica. Durante el período prepuberal se alternan, con intervalos de aproximadamente dos años, períodos de crecimiento rápido y de crecimiento más lento ${ }^{13}$ (figura 5).
Otro aspecto a considerar es la existencia de períodos normales de deceleración de la VC: durante los primeros 12-18 meses de vida en los niños con una talla baja constitucional o un tempo madurativo lento, y en la fase peripuberal en los niños con tempo madurativo lento (depresión prepuberal de la VC) $)^{14}$

\section{Talla diana (TD) o talla genética}

Talla que cabría esperar para el hijo de una determinada pareja, asumiendo que el proceso de herencia ha sido normal y que los factores ambientales han influido de forma similar en ambas generaciones. El método propuesto por Tanner es el más empleado:

- Niños: (talla padre + talla madre) / $2+6,5 \mathrm{~cm}( \pm 10)$.

- Niñas: (talla padre + talla madre) / $2-6,5 \mathrm{~cm}( \pm 9)$.

\section{Clasificación de la talla baja}

Se distinguen dos categorías principales: niños normales con talla baja y niños con talla baja secundaria a una causa patológica ${ }^{15}$. En el primer caso se encuentran los niños con talla baja familiar y con retraso constitucional del crecimiento y desarrollo y la pubertad. El segundo grupo incluiría a todos aquellos niños en los que exista una anoma- 
Figura 5. Patrón de crecimiento prepuberal en un niño con crecimiento normal.
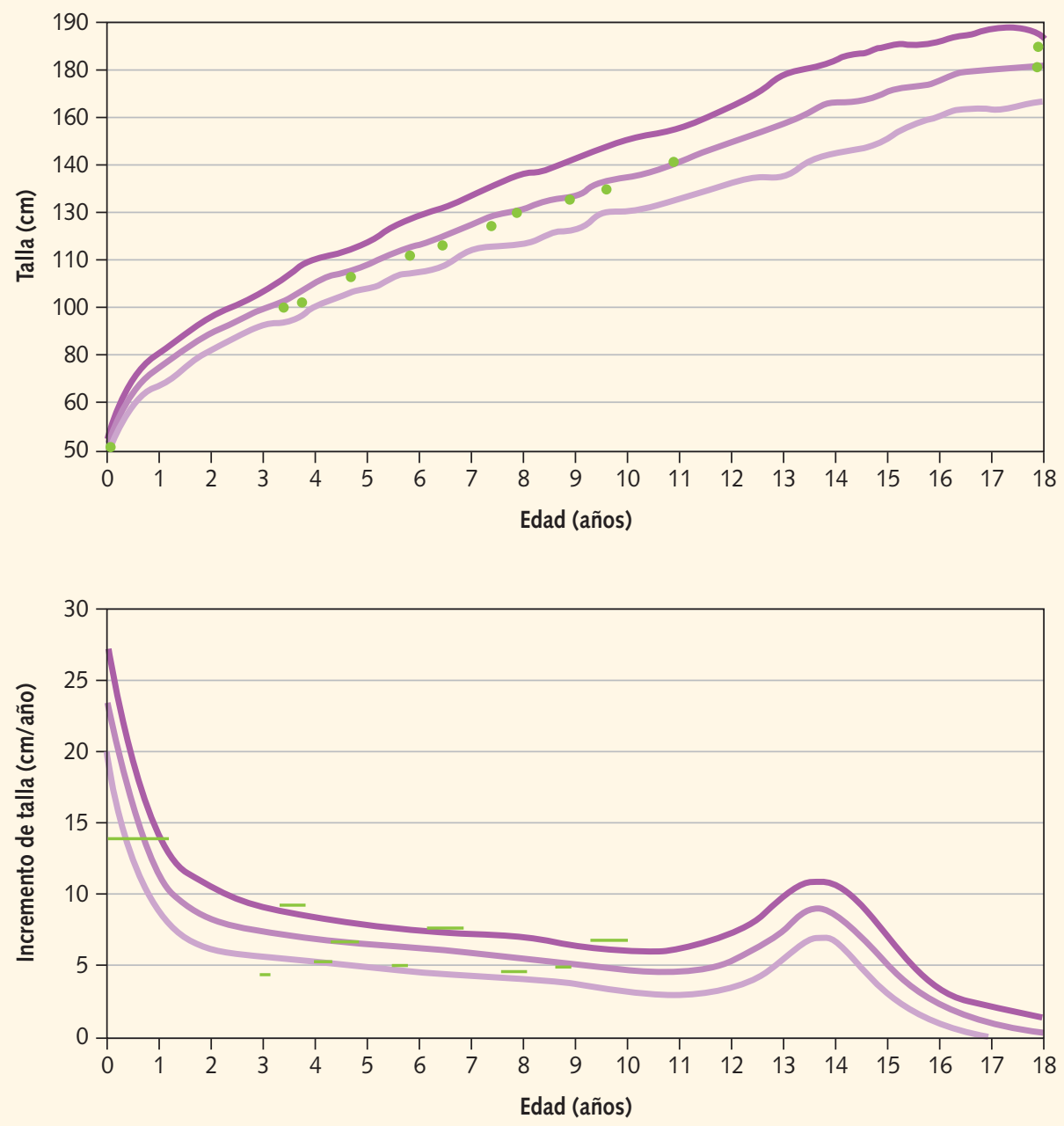

lía, demostrable o no, que sea la causa de su talla baja (tabla I).

\section{Diagnóstico}

Para llegar al diagnóstico se debe realizar:

\section{Historia clínica}

Antecedentes familiares: tallas de los padres, edad de la menarquia de la madre, afeitado o cambio de voz en el padre. Existencia de tallas bajas o altas en los abuelos y tíos, así como retraso o 


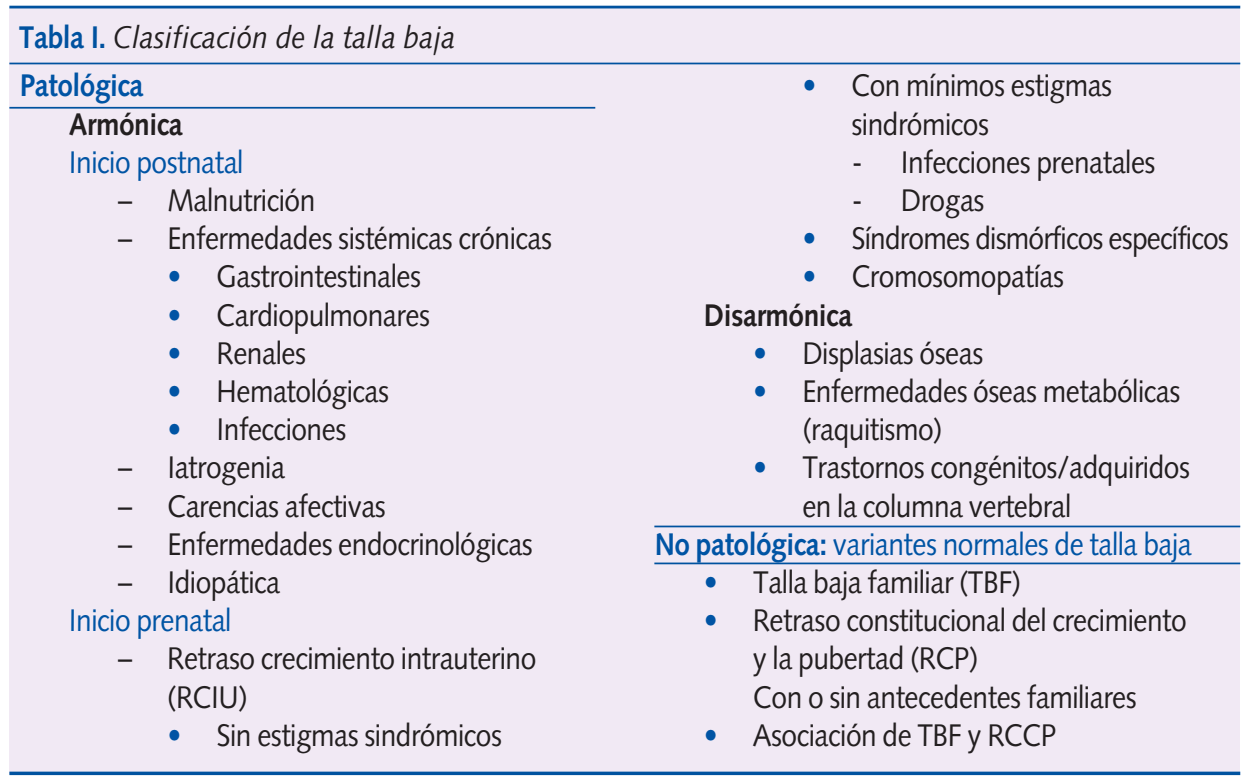

Figura 6. Deformidad de Madelung. Acortamiento e incurvación del radio con subluxación dorsal del extremo distal del cúbito.

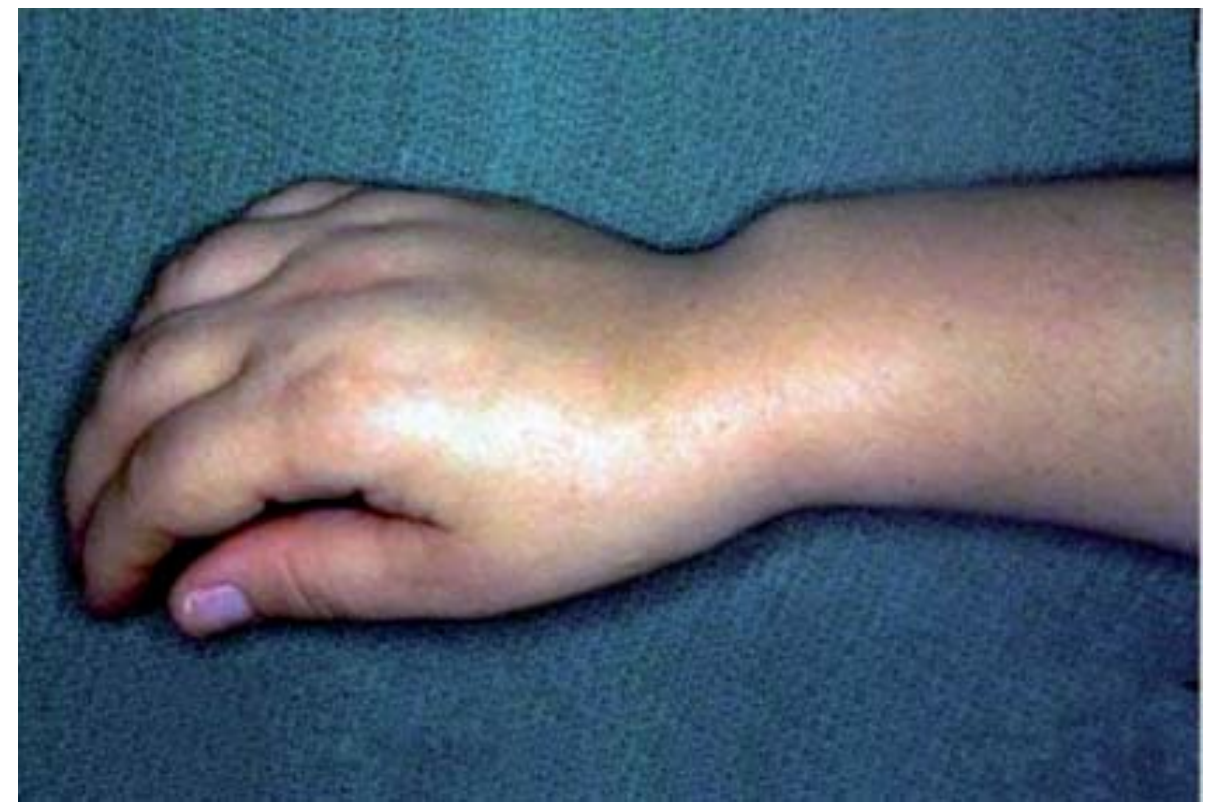


adelanto de la pubertad. Características somáticas de los padres como cortedad de extremidades, un segmento inferior especialmente corto, hábito corporal robusto, deformidad de Madelung (figura 6) (acortamiento e incurvación del radio con subluxación dorsal del extremo distal del cúbito, que se asocia a anomalías del gen SHOX), etc., apuntan a que estas alteraciones son las responsables de la talla baja y casi siempre son más evidentes en la edad adulta.

Antecedentes personales: embarazo, edad gestacional, si el parto fue normal o presentó asfixia perinatal, el peso y la talla neonatal para descartar retraso de crecimiento intrauterino (RCIU). La VC es un parámetro básico, se debe documentar siempre que sea posible y si no, se debe esperar 6 meses a valorarla antes de iniciar el estudio.

\section{Exploración}

Exploración física: permite clasificar la talla baja en armónica o disarmónica, especialmente relacionada esta última con las displasias óseas y los síndromes dismórficos.

El peso, la talla, el perímetro craneal y la VC sirven para valorar el crecimiento. La relación peso/talla, los pliegues cutáneos y el perímetro de cintura valoran el estado de nutrición.
Los niños de 0 a 2 años deben medirse en decúbito supino (longitud) y a partir de esta edad de pie (talla), ya que es así como se han establecido las tablas de referencia. La diferencia media entre ambas medidas en un niño de 18 a 20 meses es de 1-2 cm a favor de la talla en decúbito.

Valoración de la maduración: se entiende por maduración el conjunto de cambios que presenta un individuo durante su infancia y adolescencia hasta alcanzar el máximo potencial de crecimiento y desarrollo. Los indicadores más usados para valorar el estado madurativo son: la aparición y evolución de los caracteres sexuales secundarios y la maduración esquelética.

1. Estadios de Tanner ${ }^{16}$. En las niñas se valoran las mamas y el vello pubiano. En los niños el aspecto global de los genitales externos, el vello pubiano y el volumen testicular. El inicio de la pubertad en las niñas viene marcado por la aparición del botón mamario y en los niños el aumento de volumen testicular (> $4 \mathrm{cc}$ ), la aparición aislada de vello pubiano no es un indicio de inicio de la pubertad.

\section{Maduración esquelética (edad ósea).}

El grado de osificación se presenta como un indicador relativamente preciso para medir la edad fisiológica. La zona anatómica más empleada es la mano (mano iz- 
quierda por consenso internacional) por las siguientes razones: fácil accesibilidad, escasa radiación y la existencia de un amplio número de huesos en una pequeña zona corporal.

- Atlas de Greulich y Pyle ${ }^{17}$ para la mano y muñeca. Se basa en la comparación de una radiografía problema con una serie de radiografías estándares, tomadas de una muestra de la población general. Se adscribe la edad ósea que corresponda al estándar más parecido o una edad intermedia entre dos estándares sucesivos. Es la forma más utilizada. En nuestro medio Hernández y cols. ${ }^{18}$ han elaborado un atlas siguiendo los criterios de Greulich y Pyle, pero con una muestra de población española.

- Método de Tanner y Whitehou$\mathbf{s e}^{19}$. Tanner et al. describieron tres métodos de maduración ósea, utilizando la mano y muñeca: carpo (emplea solamente los 7 núcleos del carpo), RUS (13 núcleos: radio, cúbito y los huesos cortos de la mano) y 20 huesos (los 7 del carpo más los 13 del RUS), siendo el RUS el más empleado. Es un método numérico que describe una serie de indicadores de maduración para cada núcleo de osificación y asignan una puntuación a cada uno de los estadios evolutivos según el sexo. Una vez obtenida la suma de las puntaciones el resultado se traslada a una tabla que nos da la edad ósea.

- Método SHS ${ }^{20}$. En el primer año de vida no son valorables los métodos anteriores, ya que al nacimiento no existe ningún núcleo de osificación en la radiografía de la mano y estos van apareciendo a lo largo del primer año. El método SHS, desarrollado por Hernández y cols. se basa en una radiografía lateral del pie y tobillo izquierdos. Es un método numérico y valora cinco núcleos de osificación (calcáneo, cuboides, tercera cuña y las epífisis distales de tibia y peroné) a los que se les da una determinada puntuación dependiendo de los criterios madurativos que cumplan. La suma de todos ellos nos dará la maduración ósea, que compararemos con los estándares de la población general.

La diferencia entre la edad ósea y la edad cronológica en \pm un año debe considerarse normal.

Predicción de talla adulta: más importante que la talla actual de un niño, para valorar si esa talla baja es patológi- 
ca o no, es la talla definitiva que va alcanzar. Se han desarrollado diversos métodos para calcularla:

- Método de Bayley-Pinneau' ${ }^{21}$. Se basa en la alta correlación que existe entre el porcentaje de la talla adulta alcanzado y la edad ósea determinada por el método de Greulich y Pyle. El atlas de edad ósea proporciona unas tablas con el porcentaje de talla alcanzado para cada edad ósea que permiten el cálculo.

- Método de Tanner-Whitehouse (Mark II TW2)22. Se basa en ecuaciones de regresión múltiple. La edad ósea se calcula con el método RUS-TW2. Las variables incluidas son: edad ósea, talla, edad cronológica, e incremento de la talla e incremento de la edad ósea en el último año.

\section{Determinaciones analíticas}

1. Generales, que deben incluir: hemograma, estudio de la función hepática y renal, hierro, ferritina, anticuerpos antitransglutaminasa $A$ e inmunoglobulina $A$, encaminadas a excluir cualquier enfermedad crónica causante del retraso de crecimiento.

2. Hormonales, entre las que se incluyen:
- Hormonas tiroideas: TSH y T4 libre.

- Cortisol libre urinario si se asocia obesidad.

- Factores de crecimiento dependientes de la hormona de crecimiento y sus proteínas trasportadoras: IGF-1 e IGFBP-3, su utilidad se debe a que su vida media es más larga que la de la $\mathrm{GH}$ y a que sus valores se correlacionan. Los valores de IGF-1 están disminuidos en los estados de malnutrición y varían con la edad y estadio pubera ${ }^{16}$.

- La secreción pulsátil de la hormona de crecimiento no permite su valoración con una determinación aislada, por lo que es necesario realizar pruebas de provocación. Se considera normal la respuesta $>10$ $\mathrm{ng} / \mathrm{ml}$. Son necesarias dos pruebas negativas para el diagnóstico de déficit de hormona de crecimiento, debido a que el estrés u otros factores pueden inducir una liberación de hormona de crecimiento y bloquear la respuesta posterior a los estímulos farmacológicos. En el período peripuberal, así como en la obesidad las respuestas son menores, dificultando el diagnóstico ${ }^{15}$.

Se han desarrollado múltiples pruebas de provocación, las más utilizadas son: hipoglucemia inducida por insulina, clo- 
nidina, arginina, glucagón, ejercicio y ejercicio + propanol, GHRH.

\section{Técnicas de imagen}

Radiografía de mano izquierda: para la correcta valoración de la edad ósea es importante una buena técnica de la radiografía; la palma de la mano izquierda apoyada en la placa, el eje del dedo medio alineado con el antebrazo estando este en el mismo plano horizontal que el brazo, los dedos no se deben tocar, y el pulgar debe situarse de forma natural formando un ángulo de $30^{\circ}$.
Resonancia magnética nuclear craneal de la zona hipotálamo-hipofisaria. Las anomalías más frecuentes en el déficit de hormona de crecimiento son la hipoplasia de la adenohipófisis con o sin sección del tallo hipofisario, la neurohipófisis ectópica y la silla turca vacía.

\section{Estudios genéticos}

Cariotipo para descartar síndromes asociados a talla baja (obligado en las niñas para descartar síndrome de Turner).

Estudios de genética molecular están indicados en los casos con déficit idiopático de $\mathrm{GH}$ en los que existan otros casos

Figura 7. Algoritmo diagnóstico de talla baja.

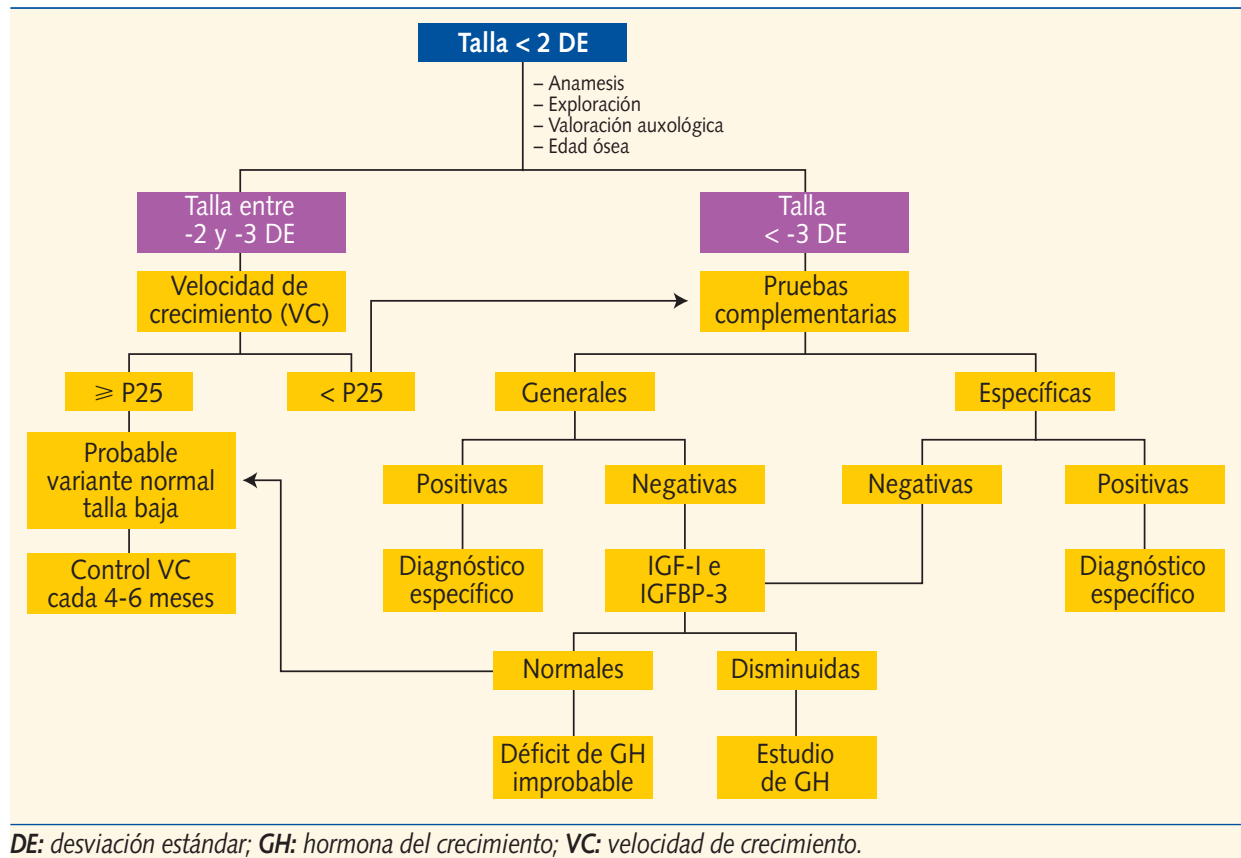


familiares; en los casos en que se sospechen alteraciones en los receptores de $\mathrm{GH}$, como cuando se encuentra IGF-1 e IGFBP-3 bajas con GH normal o elevada; en los casos de tallas bajas familiares con deformidad de Madelung en la madre (anomalía del gen $\mathrm{SHOX}^{23}$. Estas anomalías se transmiten en general de forma autosómica recesiva ${ }^{15}$.

A pesar de todas las pruebas complementarias, los criterios auxológicos siguen siendo los más importantes para orientar el estudio y el diagnóstico (figura 7).

Si la talla es baja entre -2 y -3 DE con edad ósea normal o retrasada y pronóstico de talla final igual a la talla genética con velocidad de crecimiento normal, probablemente se trata de una variante normal de talla baja.

\section{Criterios auxológicos de las variantes} normales de talla baja

Talla baja familiar (TBF) inicio del hipocrecimiento en la primera infancia, existencia de tallas bajas familiares, ritmo de crecimiento lento hasta los 2-3 años, maduración normal con inicio de la pubertad normal, edad ósea igual a la edad cronológica y mayor que la edad talla. Pronóstico de talla igual a la talla genética. La talla final será baja.

Retraso constitucional del crecimiento y la pubertad (RCCP), inicio del hipocre- cimiento en la primera infancia, historia familiar de maduración tardía con tallas normales, ritmo de crecimiento lento hasta los 2-3 años y peripuberal, maduración lenta con inicio de la pubertad tardío, la edad ósea menor que la edad cronológica e igual a la edad talla. Pronóstico de talla igual a la talla genética. La talla final será normal.

Talla baja familiar más retraso constitucional del crecimiento y la pubertad $(\mathrm{TBF})+(\mathrm{RCCP})$, inicio del hipocrecimiento en la primera infancia, historia familiar de maduración tardía con tallas bajas, ritmo de crecimiento lento hasta los 2-3 años y peripuberal, maduración lenta con inicio de la pubertad tardío, la edad ósea menor que la edad cronológica y mayor que la edad talla. Pronóstico de talla igual a la talla genética. La talla final será baja.

Si la talla es muy baja, menor de -3 DE, con edad ósea retrasada o el pronóstico de talla final es menor que la talla genética o la VC es baja son precisos más estudios para descartar una causa patológica de talla baja.

\section{Criterios auxológicos de algunas variantes patológicas de talla baja}

Talla baja por déficit de $\mathrm{GH}$ : peso y talla normales al nacimiento, VC disminuida, retraso de edad ósea mayor de 
Tabla II. Hipocrecimientos de causa conocida que pueden diagnosticarse erróneamente de "variante normal de talla baja" o de "talla baja idiopática"

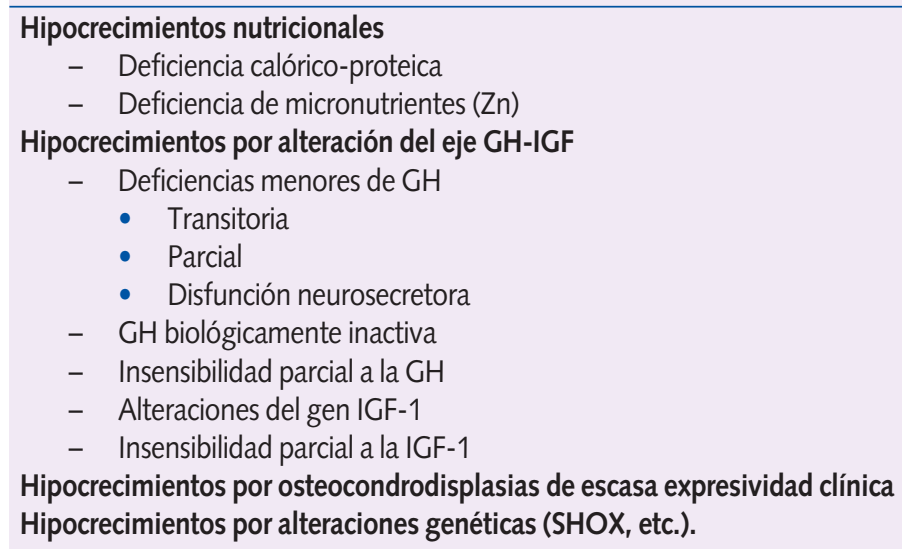

un año. Pronóstico de talla inferior a la talla genética.

Talla baja en el RCIU: peso y/o talla baja al nacimiento, -2,5 DE para su edad de gestación, se recomienda utilizar las tablas de recién nacidos del estudio transversal español. Falta de crecimiento recuperador con talla a los 4 años menor de 2,5 DE y/o menor de -1 DE, ajustada a la talla media parental. Edad ósea retrasada alrededor de 2 años en el período prepuberal, con maduración rápida en el período puberal. La talla final será baja.

Finalmente, por exclusión de todos los diagnósticos anteriores llegaremos a la talla baja idiopática, donde probablemente estén englobados niños con talla baja sin patología asociada realmente y niños con patología asociada en los que, o bien no se ha llegado al diagnóstico (tabla II) o todavía no conocemos la causa $^{15}$.

\section{Tratamiento}

En el caso de déficit de hormona de crecimiento el tratamiento será hormona de crecimiento a dosis sustitutivas. Sin embargo, existen otras patologías sin déficit de hormona de crecimiento en las que se ha demostrado su utilidad, si bien en general, a dosis mayores. Ambos tratamientos son bien tolerados. Ocasionalmente puede aparecer hipertensión craneal benigna (dolor de cabeza, náuseas y/o vómitos y edema de papila). Igualmente existe riesgo de hiperglucemia y glucosuria. Se han descrito casos de desplazamiento epifisario de la cabeza del fémur, detectado por la aparición de cojera. 
Actualmente las indicaciones de tratamiento de talla baja con hormona de crecimiento autorizadas en España son ${ }^{24}$ :

- Defecto de hormona de crecimiento a una dosis de 0,025-0,035 $\mathrm{mg} / \mathrm{kg} / \mathrm{día}$, con los siguientes criterios de inclusión: talla inferior a 2 DS para su edad o por debajo de 1 DS para la talla media paterna, VC por debajo del P10, edad ósea retrasada más de 1 año, dos test farmacológicos de secreción de $\mathrm{GH}$ que no respondan ( $\mathrm{GH}$ inferior a 10 $\mathrm{ng} / \mathrm{ml}$ ). En el seguimiento se deben realizar determinaciones de T4 libre e IGF-1 e IGFBP-3, en algunos casos es necesario asociar al tratamiento L-tiroxina por la presencia de un hipotiroidismo subyacente no evidenciado previamente.

- Síndrome de Turner a partir de los 2 años de vida a una dosis de 0,035-0,40 mg/kg/día s.c., cuando exista una VC por debajo P10 para su edad ósea durante 6 meses. En el seguimiento se deben realizar determinaciones de T4 libre y anticuerpos antitiroideos (ATA), IGF-1 e IGFBP-3, hemoglobina glicosila$\mathrm{da}$, y marcadores de enfermedad celíaca.

- Insuficiencia renal crónica (IRC) a partir de los 2 años a una dosis de
0,045-0,050 mg/kg/día. Con los siguientes criterios de inclusión IRC (filtrado glomerular inferior al 50\%), tratamiento crónico de diálisis, situación prepuberal, talla inferior a 2 DS para su edad o por debajo de 1 DS para la talla media paterna, velocidad de crecimiento por debajo del $\mathrm{P} 10$, edad ósea retrasada más de 1 año. En el seguimiento realizar T4 libre e IGF-1 e IGFBP-3. Criterios de exclusión: patología cardiovascular severa, osteopatía severa, diabetes mellitus, enfermedad maligna activa y trasplante renal.

- Síndrome de Prader Willi a partir de los 3 años a una dosis de 0,35 a 1 $\mathrm{mg} / \mathrm{kg} /$ día, máximo $2,7 \mathrm{mg} /$ día, cuando exista retraso de maduración ósea en más de un año. En el seguimiento se deben realizar determinaciones de T4 libre, IGF-1 e IGFBP-3, hemoglobina glicosilada, composición corporal y radiografía de columna dorso-lumbar posteroanterior, ya que una complicación grave es la presentación de escoliosis. Criterios de exclusión: obesidad mórbida (peso > 150\% peso ideal), intolerancia a la glucosa o diabetes mellitus, escoliosis $\geqslant 20^{\circ}$, apnea del sueño, hipertrofia obstructiva amígdalo-adenoidea. 
- Crecimiento intrauterino retardado a partir de los 4 años si la talla es inferior a -2,5 DE o menor de -1 DE ajustada a la talla parental a una dosis de 0,035-0,067 mg/kg/día subcutáneo. En el seguimiento se deben realizar determinaciones de T4 libre, IGF-1 e IGFBP-3, glucemia e insulinemia basal, hemoglobina glicosilada, lipidograma y presión arterial. Criterios de exclusión son el inicio de la pubertad, niños con síndrome de Silver Russell o cualquier otro, intolerancia a la glucosa o diabetes mellitus, resistencia insulínica, pacientes tratados con análogos de GnRH.

- Deficiencia de crecimiento debida a alteración del gen SHOX, a partir de los 2 años con talla inferior a $2 \mathrm{DE}, \mathrm{VC}$ disminuida por debajo de P10 para su correspondiente edad ósea, mantenida durante un mínimo de 6 meses. A una dosis de 0,045-0,050 mg/kg/día. En el seguimiento se realizará T4 libre, IGF-1 e IGFBP3, hemoglobina glicosilada.

La FDA aprobó en 2003 el tratamiento con hormona de crecimiento en niños con talla baja idiopática y talla baja familiar con una talla inferior a 2,25 DE en el momento de inicio del tratamiento y un pronóstico de talla inferior a $160 \mathrm{~cm}$ en hombres y a $150 \mathrm{~cm}$ en mujeres. Dado que se trata de un grupo heterogéneo de pacientes las repuestas al tratamiento son muy diferentes. Los estudios realizados muestran un aumento de la VC a corto plazo, y en aquellos que muestran talla final con una ganancia media de 4 a $6 \mathrm{~cm}$ $(+0,84 \mathrm{DE})^{25}$ la talla final sigue siendo baja $^{26}$. Otros tratamientos que se utilizan en las tallas bajas idiopáticas y familiares son los análogos de GnRH con el intento de mejorar la talla final retrasando la pubertad y el cierre de las epífisis. La ganancia sobre la talla prevista varía entre 0 y 4 $\mathrm{cm}^{27}$. En la actualidad existen estudios en marcha utilizando un tratamiento que combina hormona de crecimiento con análogos de $\mathrm{GnRH}^{28}$ que parece pueden conseguir mejores resultados.

\section{Conclusión}

En el diagnóstico de talla baja son fundamentales los criterios auxológicos, entre los que destaca la VC para considerar una talla baja normal o patológi$\mathrm{ca}$, sin embargo existen grandes variaciones en la misma, por lo que se deben elegir los intervalos adecuados. Existe gran controversia en cuanto al tratamiento de las formas idiopáticas y de aquellas variantes familiares consideradas normales. 


\section{Bibliografía}

1. Tanner JM. Foetus into man. Physical growth from conception to maturity (2nd ed.) Cambridge MA: Harvard University Press; 1989.

2. Karlberg J. A biologically-oriented mathematical model ICP for human growth. Acta Paedriatr Scand Suppl. 1989;Supl 350:s74-s94.

3. Sobradillo B, Aguirre A, Aresti U, Bilbao C, Fernández-Ramos $C$, Lizarraga $A$, et al. Curvas y tablas de crecimiento (estudio longitudinal $y$ transversal). Fundación F. Orbegozo, En: Patrones de crecimiento y desarrollo en España. Atlas de gráficas y tablas. Madrid: Ergon; 2004. p. 145-68.

4. Ferrández-Longás $A$, Mayayo E, Labarta Jl, Bagué L, Puga B, Rueda $C$, et al. Estudio longitudinal de crecimiento y desarrollo. Centro Andrea Prader. Zaragoza 1980-2002. En: Patrones de crecimiento y desarrollo en España. Atlas de gráficas y tablas. Madrid: Ergon; 2004. p. 61-115.

5. Carrascosa A, Copi A, Yeste B, Gussigüe M. Curvas de crecimiento. Barcelona. En: Patrones de crecimiento y desarrollo en España. Atlas de gráficas y tablas. Madrid: Ergon; 2004.

6. Carrascosa Lezcano A, et al. Estudio transversal español de crecimiento 2008 (Parte II). An Pediatr (Barc). 2008;68(6):552-69.

7. Carrascosa Lezcano A, Fernández García $J M$, Fernández Longás A, López-Siguero JP, Sánchez González E. Estudio transversal español de crecimiento 2008. Pfiizer 2008.

8. WHO Multicentre Growth Reference Study Group. WHO Child Growth Standards based on length/height, weight and age. Acta Paediatr Suppl. 2006;450:76-85.

9. WHO Working Group on the Growth Reference Protocol and WHO Task Force on Methods for the Natural Regulation of Fertility. Growth patterns of breastfed infants in seven countries. Acta Paediatr. 2000;89:215-22.
10. WHO Multicentre Growth Reference Study Group. Assessment of differences in linear growth among populations in the WHO Multicentre Growth Reference Study. Acta Paediatr Suppl. 2006;450:56-65.

11. Hermanussen M. The analysis of shortterm growth. Horm Res. 1998;49:53-64.

12. Wehr TA. Effect of seasonal changes in daylength on human neuroendocrine function. Horm Res. 1998;49(3-4):118-24.

13. Butler GE, McKie M, Ratcliffe SG. The cyclical nature of prepubertal growth. Ann Hum Biol. 1990;17:177-98.

14. Smith DW, Truog W, Rogers JE, Greitzer LG, Skinner AL, McCann JJ, Harvey MAS. Shifting linear growth during infancy and the genetics of growth and fetal life through infancy. J Pediatr. 1976;89:225-30.

15. Pozo J, Gracia R, Argente J. Hipocrecimiento: concepto, clasificación y metodología diagnóstica. En: Argente J, Carrascosa A, Gracia $\mathrm{R}$, Rodríguez $\mathrm{F}$ (eds.). Tratado de endocrinología pediátrica y de la adolescencia (2. ${ }^{\text {a }}$ ed.). Madrid: Doyma; 2000. p. 201-45.

16. Tanner JM. Foetus into man. Physical growth from conception to maturity (2nd ed.) Cambridge MA: Harvard University Press; 1989.

17. Greulich WW, Pyle SI. Radiographic atlas of skeletal development of the hand and wrist (2nd ed.). Stanford: Stanford University Press; 1959.

18. Hernández M, Sánchez E, Sobradillo $B$, Rincón JM. Maduración ósea y predicción de talla adulta. Atlas y métodos numéricos. Madrid: Ed. Díaz de Santos; 1991.

19. Tanner JM, Whitehouse RH, Marshall WA, Healy MJR, Goldstein H. Assessment of skeletal maturity and prediction of adult height (TW2 method) (2nd ed.). Londres: Academic Press; 1983. 
20. Hernández $M$, Sánchez $E$, Sobradillo $B$, Rincón JM, Narvaiza JL. A new method for assessment of skeletal maturity in the first 2 years of life. Pediatr Radiol. 1988;18:484-9.

21. Bayley N, Pinneau S. Tables for predicting adult height from skeletal age. J Pediatr. 1952; 14:423-41.

22. Tanner JM, Landt KW, Cameron N, Carter BS, Patel J. Prediction of adult height from height and bone age in childhood. A new system of equations (TW Mark II) based on a sample including very tall and very short children. Arch Dis Child. 1983;58:767-76.

23. Rappold G, Blum WF, Shavrikova EP, et al. Genotypes and phenotypes in children with short stature: clinical indicators of SHOX haploinsufficiency. J Med Genet. 2007;44:306.

24. Criterios para la utilización racional de la hormona de crecimiento en niños. Disponible en www.msc.es/profesionales/farmacia/documentacion.htm
25. Finkelstein BS, Imperiale TF, Speroff $T$, Marrero U, Radcliffe DJ, Cuttler L. Effect of growth hormone therapy on height in children with idiopathic short stature: a meta-analysis. Arch Pediatr Adolesc Med. 2002;156(3):230-40.

26. Bryant J, Baxter L, Cave CB, Milne R. Recombinant growth hormone for idiopathic short stature in children and adolescents. Cochrane Database of Systematic Reviews 2005, Issue 4. Art. No.: CD004440. DOI: 10.1002/14651858. CD004440.pub2

27. Yanovski JA, Rose SR, Municchi G, Pescovitz OH, Hill SC, Cassorla FG, Cutler GB Jr. Treatment with a luteinizing hormone-releasing hormone agonist in adolescents with short stature. N Engl J Med. 2003 Mar 6;348(10):908-17.

28. Castro-Feijóo L, Castro-Feijóo C, Quinteiro García J, Campbell Cruz J, Barreiro Conde. M Pombo Arias. Tratamiento con análogos de $\mathrm{GnRH}$ y $\mathrm{GH}$ en niños con talla baja familiar. An Pediatr (Barc). 2002;56:106-12. 